(D) Check for updates

Cite this: Food Funct., 2019, 10, 514

\title{
The effects of polyphenols and other bioactives on human health
}

\author{
César G. Fraga, (D)*a,b,c Kevin D. Croft, (D) d David O. Kennedy and \\ Francisco A. Tomás-Barberán (D) ${ }^{f}$
}

\begin{abstract}
Although deficiencies in polyphenol intake do not result in specific deficiency diseases, adequate intake of polyphenols could confer health benefits, especially with regard to chronic diseases. Tea, cocoa, fruits, and berries, as well as vegetables, are rich in polyphenols. Flavan-3-ols from cocoa have been found to be associated with a reduced risk of stroke, myocardial infarction, and diabetes, as well as improvements in lipids, endothelial-dependent blood flow and blood pressure, insulin resistance, and systemic inflammation. The flavonoid quercetin and the stilbene resveratrol have also been associated with cardiometabolic health. Although polyphenols have been associated with improved cerebral blood flow, evidence of an impact on cognition is more limited. The ability of dietary polyphenols to produce clinical effects may be due, at least in part, to a bi-directional relationship with the gut microbiota. Polyphenols can impact the composition of the gut microbiota (which are independently associated with health benefits), and gut bacteria metabolize polyphenols into bioactive compounds that produce clinical benefits. Another critical interaction is that of polyphenols with other phytochemicals, which could be relevant to interpreting the health parameter effects of polyphenols assayed as purified extracts, whole foods, or whole food extracts.
\end{abstract}

Received 12th October 2018, Accepted 5th January 2019

DOI: $10.1039 / \mathrm{c} 8 \mathrm{fo} 01997 \mathrm{e}$

rsc.li/food-function fiber, carotenoids, and phytosterols, that could promote health. In particular, the class of phytochemicals called polyphenols may confer health benefits, particularly as they relate to NCDs. ${ }^{4-7}$ In contrast to vitamins and minerals and other nutrients, inadequacies in plant polyphenol intake are not associated with specific deficiency diseases, making it difficult to define appropriate reference intake values for these food components. ${ }^{8}$ Despite this knowledge gap, many of these compounds have been identified, with some producing promising results in observational studies and randomized clinical trials in certain NCDs. ${ }^{9-11}$ The purpose of this narrative review is to explore how polyphenols exert health benefits in humans, alone or in synergy with other plant components.

\section{The absorption and metabolism of polyphenols}

${ }^{a}$ Fisicoquimica, Facultad de Farmacia y Bioquimica, Universidad de Buenos Aires, Buenos Aires, Argentina.E-mail: cgfraga@ucdavis.edu, cfraga@ffyb.uba.ar; Tel: +54-11-5287 4237

${ }^{b}$ Instituto de Bioquímica y Medicina Molecular (IBIMOL), UBA-CONICET, Buenos Aires, Argentina

${ }^{c}$ Department of Nutrition, University of California, Davis, CA, USA

${ }^{d}$ School of Biomedical Science, University of Western Australia, Perth, Western Australia

${ }^{e}$ Brain Performance and Nutrition Research Centre, Northumbria University, Newcastle Upon Tyne, UK

${ }^{f_{S p a n i s h}}$ National Research Council, CEBAS-CSIC, PO Box 164, Espinardo, Murcia 30100, Spain
The polyphenols constitute a large group of bioactive phytochemicals that include multiple sub-classes such as flavonoids, stilbenes, phenolic acids, and lignans. ${ }^{12}$ Fig. 1 describes the basic structure of some key classes of these phenolic compounds, as well as some other bioactive phytochemicals, such as terpenoids (e.g. carotenoids) and nitrogen- (e.g. alkaloids) and sulfur-containing products (e.g. sulforaphanes, allicin). ${ }^{13}$ One of the most studied groups of polyphenols is the flavonoids, which are structurally based on a 15-carbon skeleton of 


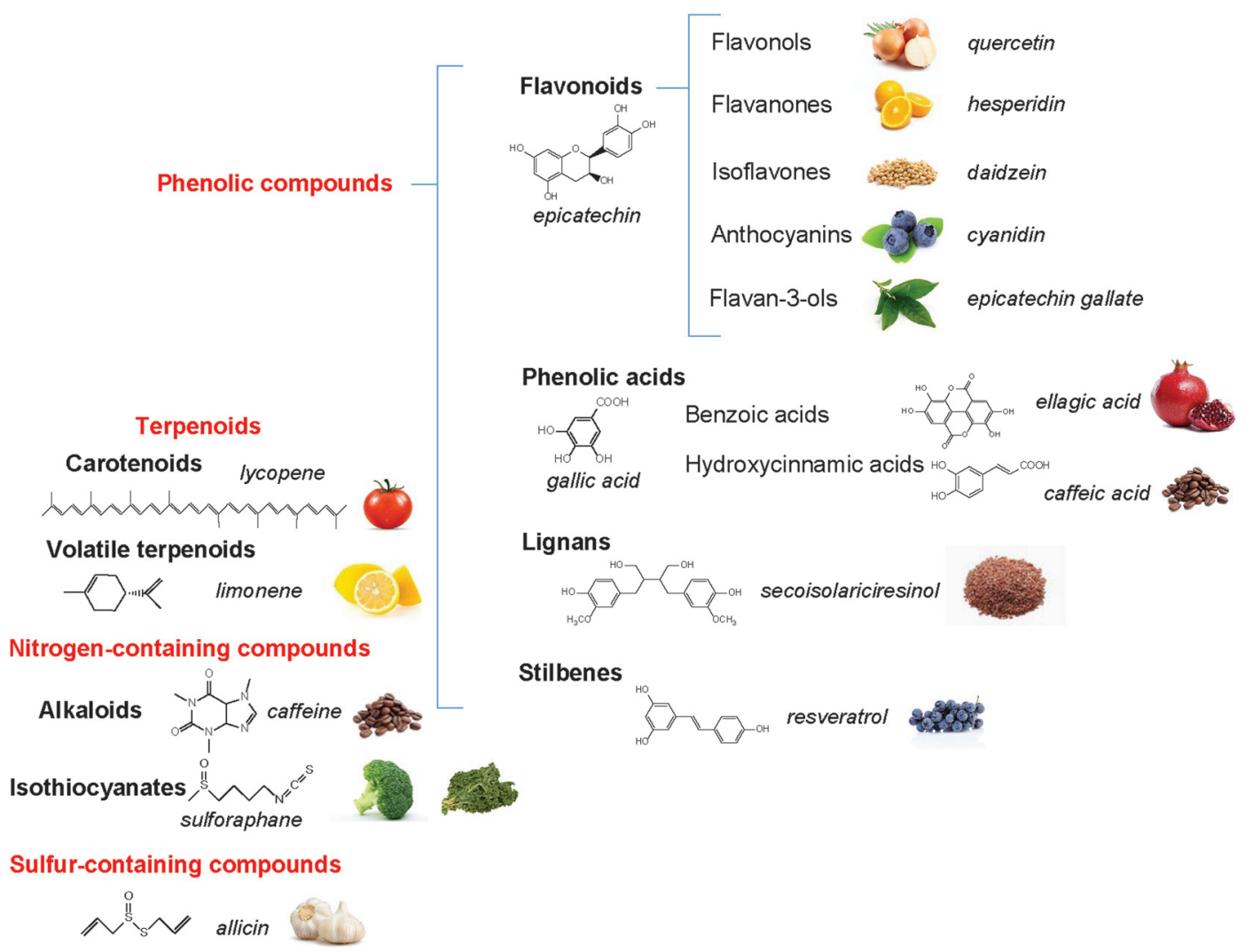

Fig. 1 Structure of common phytochemicals.

a chromane ring attached to a second aromatic ring (Fig. 2). The flavonoids can be sub-divided into groups, including flavan-3-ols, flavonols, flavones, isoflavones, flavanones, and anthocyanins. Table 1 provides specific examples of these natural polyphenols and describes some known food sources for each class. ${ }^{14-16}$

Naturally occurring flavonoids are stored in plants as glycoside and non-glycosylated conjugates, and the nature of the moiety can influence their later bioavailability in humans. ${ }^{12,17}$ Flavonoids can be absorbed in the small intestine, where they are frequently metabolized by phase II enzymes before entering systemic circulation. ${ }^{17,18}$ Although a proportion of some dietary flavonoids are absorbed in the small intestine, a significant amount enters the large intestine where the colonic microbiota further degrade the deconjugated metabolites and aglycones into readily absorbable molecules, including pheno-<smiles>C1=C(c2ccccc2)Oc2ccccc2C1</smiles>

Fig. 2 Basic structure of flavonoids. lic acids. ${ }^{18}$ For example, tomato juice quercetin-3-O-rutinosides are primarily absorbed in the large rather than the small intestine, and there are multiple methylated and glucuronidated products that are metabolized by colonic microflora. ${ }^{17}$ Green tea flavan-3-ols are also extensively metabolized prior to being absorbed, initially in the small intestine; a remaining portion enters the large intestine and is metabolized by the microbiota to form phenolic acids. ${ }^{17}$ The available data indicate that colonic bacteria play a significant role in the absorption of a host of flavonoids, as well as other phenolic and polyphenolic compounds, and can determine their bioavailability in systemic circulation. Furthermore, the presence of polyphenols and their metabolites in the colon can directly impact the health of the colon and its microbiota, as they persist in the colon for longer periods of time than in the small intestine. ${ }^{17}$

\section{Interactions between polyphenols and the microbiome}

There is a bi-directional relationship between polyphenols and the microbiome of the human gut (Fig. 3), ${ }^{11,18-21}$ that mimics to some extent the relationship between polyphenols and the pathogenic and symbiotic microbial population in the root system of plants. ${ }^{11}$ Polyphenols can modulate the composition of an individual's microbiome. ${ }^{19,21}$ For example, cocoa flavan- 
Table 1 Food sources of major polyphenol classes ${ }^{14-16}$

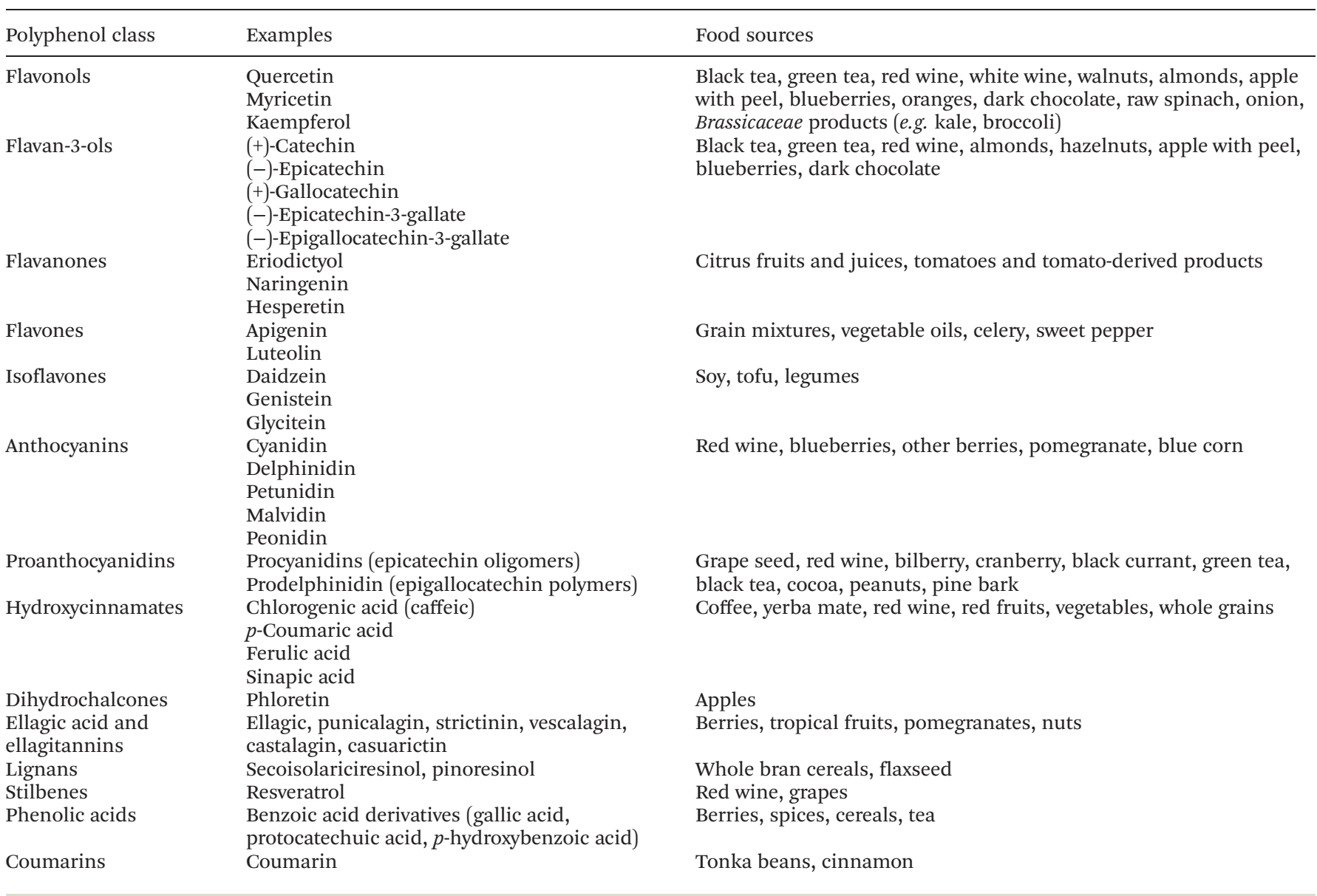

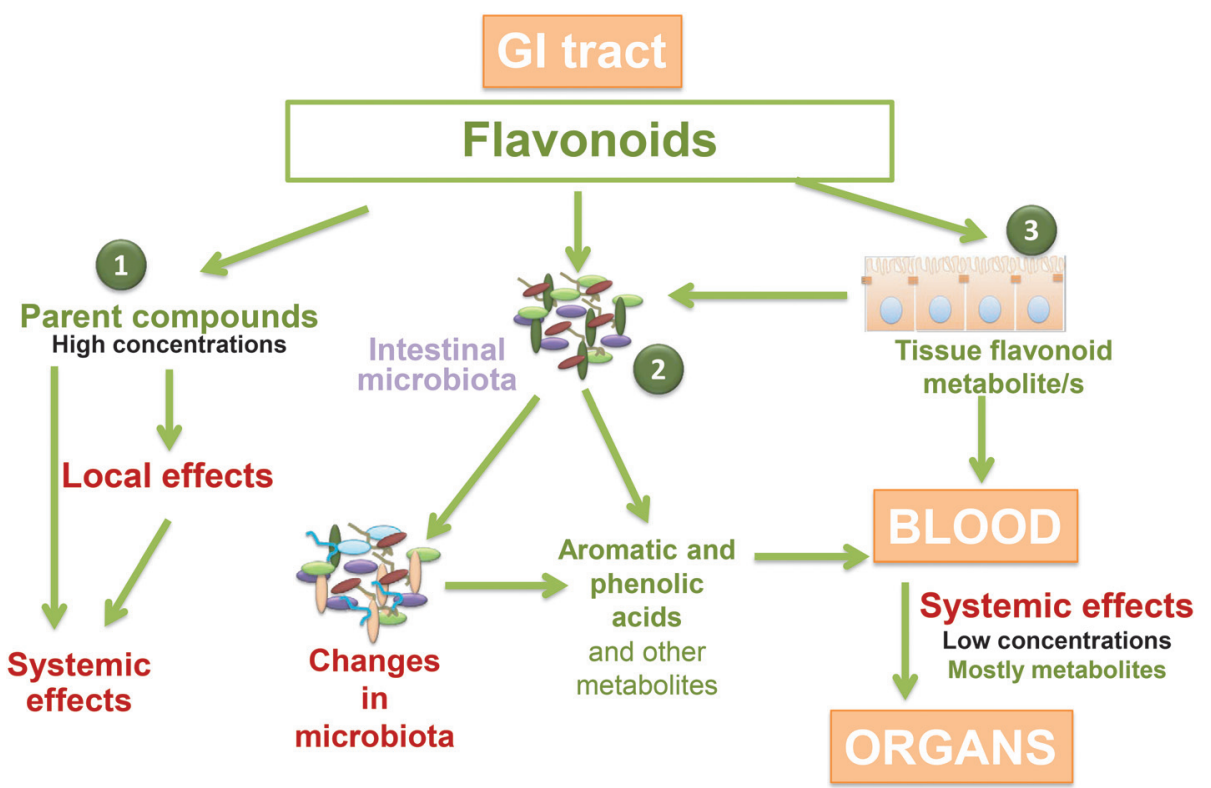

Fig. 3 Polyphenol metabolism in the colon and metabolite absorption. ${ }^{20} \mathrm{Gl}$, gastrointestinal. Reprinted from Molecular Aspects of Medicine, vol 61 , Oteiza PI, Fraga CG, Mills DA, Taft DH, Flavonoids and the Gastrointestinal Tract: Local and Systemic Effects, pp 41-49, copyright 2018, with permission from Elsevier. 
3-ols have been shown to increase the production of butyrate, and associations have been observed between fermentable polysaccharides and flavonols and flavan-3-ols. ${ }^{19}$ Non-extractable polyphenols (i.e. those that are insoluble and cannot be extracted with classical solvent extraction techniques) have been somewhat overlooked for their bioactive properties, but it has been shown that they persist in the colon for an extended period, where they are metabolized by gut microbiota to form more efficiently absorbed, active metabolites. ${ }^{22}$

The composition of an individual's gut microbiome can vary depending upon environmental and genetic factors (e.g. enterotypes, differences in diversity, and content), ${ }^{23}$ and the ability of individuals to metabolize certain polyphenols has been attributed to distinct phenotypes (i.e. metabotypes) ${ }^{24}$ For example, the population can be categorized into three distinct urolithin metabotypes of ellagitannin that have been associated with differences in intestinal microbiota, specifically Gordonibacter urolithinfaciens and Ellagibacter isourolithinfaciens. ${ }^{25-27}$ It has been suggested that the degree of ellagitannin metabolism, based on the types of bacteria that are present in the colon, can lead to differing biological effects. ${ }^{28}$ After the intake of isoflavones, a polyphenol group that has received interest for its effects in health, the conversion of daidzein to equol by the gut microbiota also shows the presence of an equol producer metabotype and an equol non-producer metabotype. These metabotypes show different distribution among individuals, and only the equol producers seem to experience cardiometabolic risk benefits after the intake of soy isoflavones. ${ }^{29}$ Since dysbiosis of gut microbiota has also been associated with the development of a number of NCDs, including cardiovascular disease, obesity, and neurodegenerative diseases,${ }^{30-32}$ a positive interaction with polyphenolic compounds has the potential to produce health benefits. ${ }^{20,21}$

Some polyphenols, such as those found in green and black tea, can inhibit the growth of detrimental bacteria such as Helicobacter pylori, Staphylococcus aureus, Escherichia coli, Salmonella typhimurium, Listeria monocytogenes, and Pseudomonas aeruginosa, as well as hepatitis C virus, influenza, HIV, and Candida. ${ }^{33}$ Other polyphenols, in contrast, can stimulate growth or at least change the composition of the microbiome in favor of beneficial bacteria including Bifidobacterium spp., Lactobacillus spp., Akkermansia muciniphila, and Faecalibacterium prausnitzii and improve the ratio of Firmicutes to Bacteroidetes. ${ }^{21,33-36}$ For example, consumption of a prebiotic wild blueberry powder drink rich in anthocyanidins for six weeks was shown to produce a statistically significant increase in Lactobacillus acidophilus and Bifidobacterium spp. compared with placebo. ${ }^{37}$

Polyphenols are metabolized by the microbiome to generate compounds with potential bioactivity. For example, chlorogenic acid and related compounds are some of the main bioactive phenolic compounds present in significant amounts in the diet. They are metabolized in the gut by the resident microbiota, which are responsible for the release of caffeic acid and further metabolism, producing phenyl-propionic, phenylacetic, and benzoic acid derivatives that are then absorbed. ${ }^{38,39}$

\section{The health benefits of polyphenols}

The primary mechanism of action of polyphenols was originally thought to lie in their direct antioxidant effects. However, these effects are no longer considered to be as relevant in vivo, as these compounds do not reach concentrations in most tissues that are high enough to have a significant effect in terms of scavenging free radicals. ${ }^{40-44}$ However, a number of other possible biochemical and molecular mechanisms have been identified, including multifarious effects within intra- and inter-cellular signaling pathways, such as regulating nuclear transcription factors and fat metabolism, and modulating the synthesis of inflammatory mediators including cytokines tumor necrosis factor $\alpha$, interleukin (IL)- $1 \beta$, and IL-6. ${ }^{45,46}$ For example, certain flavonoids have been shown to have a role in glucoregulation through downstream signaling that increases insulin secretion, reduces apoptosis, promotes $\beta$-cell proliferation, and reduces insulin resistance, inflammation, and oxidative stress in muscle and other cells. Phlorizin is a dihydrochalcone found in apples and apple products that can be absorbed in the small intestine and potentially transported by sodium-glucose transporters (SGLTs). Phlorizin is a specific and competitive inhibitor of SGLTs in the intestine (SGLT-1) and kidney (SGLT-2) and as such may be useful in treating hyperglycemia. ${ }^{47}$ As a result, flavonoids are thought to have beneficial effects on obesity and diabetes. ${ }^{4-50}$

A relevant consideration is the amounts of these compounds that need to be consumed to provide positive health effects. When the amounts of polyphenols necessary for producing health benefits are within the ranges present in commonly consumed foods, it is possible to conclude there is an absence of toxicological danger. A second level is when the potential effects are observed at levels that require an increase in the consumption of foods that is tolerable within reasonable boundaries (e.g. consuming three apples instead of two). A third level is when the amount needed requires intake levels attainable via dietary supplements or pharmacological approaches. In this latter situation, the possibilities of undesirable effects are increased, and such possibilities should be carefully controlled for.

\subsection{Effects of polyphenols on cardiometabolic health}

Polyphenols from various food sources such as cocoa, coffee, tea, and apples have been associated with various healthrelated benefits, including in cardiovascular disease and type 2 diabetes. ${ }^{4,751-64}$ Possible mechanisms include effects on blood pressure, endothelial function, glucose metabolism, inflammation, oxidative stress biomarkers, platelet function, and cholesterol, as well as indirect effects mediated by interaction with the gut microbiome..$^{20,21}$

A portion of the evidence describing the role of polyphenols in cardiovascular health comes from epidemiologic studies involving different dietary patterns and intake of certain classes of foods. For example, results from the Prevención con Dieta Mediterránea (PREDIMED) trial reported an inverse relationship between total dietary polyphenol intake from 
different sources as components of a Mediterranean Diet (e.g. red wine, nuts, and olive oil) and the risk of overall mortality. ${ }^{65,66}$ An analysis of the same study found an association between total polyphenol intake and risk of cardiovascularrelated events, independent of other dietary and non-dietary risk factors. ${ }^{66}$

Clearly, several factors need to be taken into consideration when interpreting data from observational studies. For instance, the increased consumption of polyphenol-rich foods of plant origin may be balanced by a reduced consumption of potentially detrimental foods of animal origin, and the latter may contribute to any apparent relationship between polyphenol consumption and improved function. In the case of studies involving self-reported food consumption, the accuracy of recall, differences in portion size, and potential differences in the polyphenol contents of similar foods all markedly reduce the accuracy of the estimation of polyphenol consumption and may serve to obscure any relationship. Additionally, this type of research can be confounded by other co-varying factors, and it often tells us little about causality. However, observational studies are particularly useful in the formulation of hypotheses to take forward into controlled intervention studies, and the convergence of evidence from these differing types of research is particularly persuasive. Given these factors, where observational research is cited it should be followed by consideration of the numerous clinical studies that have evaluated the health effects of administering individual phenolic compounds and phenolic-rich extracts or foods.

4.1.1. Flavan-3-ols. A number of commonly consumed foods are high in flavan-3-ols, including tea, nuts, cocoa (chocolate), grapes (wine), and legumes. ${ }^{67-69}$ Observational studies have reported that flavan-3-ol intake from various dietary sources produces positive effects on cardiometabolic outcomes, including reducing the risk for diabetes, and cardiovascular-related outcomes (i.e. cholesterol levels, blood pressure, and myocardial infarction). ${ }^{56,57,70}$ Regular chocolate intake has also been associated with a beneficial effect on arterial stiffness, which is involved in the development of systolic hypertension. ${ }^{71}$ Results from the large, prospective Cancer Prevention Study II Nutrition Cohort ${ }^{72}$ indicated that total flavonoid intake, including flavan-3-ols, was also associated with a lower risk of fatal cardiovascular disease. A metaanalysis of cohort, case-control, and cross-sectional studies reported a $37 \%$ lower risk of any cardiovascular disease (five studies), a $31 \%$ reduction in the risk of diabetes (one study), and a $29 \%$ reduced risk of stroke (three studies) in individuals who consumer higher levels of cocoa and chocolate; however, no significant effect was observed on heart failure (two studies). ${ }^{51}$ However, the individual studies reviewed did not distinguish between polyphenol-rich dark chocolate and low-polyphenol milk/white chocolate, potentially diluting and underestimating the beneficial effects of the polyphenolrich products. Meta-analyses of observational studies have also reported that consuming foods that are high in flavan-3ols can reduce the risk for stroke and myocardial infarction. ${ }^{4,53,57}$
In addition, a meta-analysis of randomized, controlled trials reported that cocoa flavan-3-ols from supplements and dietary sources produce modest but significant improvements in cardiometabolic biomarkers, including lipid metabolism, insulin resistance, and systemic inflammation. ${ }^{60}$ In a separate meta-analysis of randomized, controlled trials, dark chocolate and flavan-3-ol-rich cocoa products were shown to be superior to placebo in reducing diastolic pre-hypertension and systolic hypertension, but no significant reduction in mean systolic blood pressure $<140 \mathrm{mmHg}$ or diastolic blood pressure $<80 \mathrm{mmHg}$ was observed. ${ }^{61}$ A separate meta-analysis of randomized, controlled trials that evaluated flavonoid-rich foods, including those that are rich in flavan-3-ols, reported significant reductions in cardiovascular disease risk factors, including flow-mediated dilation (FMD). ${ }^{5}$ Furthermore, based on the results of another meta-analysis of randomized, controlled trials, different classes of flavonoids, in particular the flavan-3ols, may have differential effects on FMD and blood pressure that may be dose dependent. ${ }^{73}$ Other meta-analyses of randomized, controlled trials have investigated the effects of chocolate, cocoa, or cocoa flavan-3-ols intake and found statistically significant beneficial effects on lipid metabolism, insulin resistance, mean arterial pressure, and systemic inflammation and marginally significant effects on LDL and HDL cholesterol. ${ }^{60,62,74}$ A possible independent mechanism for these effects was proposed to be augmentation of NO status that was accompanied by enhanced endothelial function and lower systolic blood pressure. ${ }^{75,76}$

In a prospective cohort study, flavan-3-ols found in cocoa and tea have been shown to reduce the risk for cardiovascular disease mortality. ${ }^{77}$ A meta-analysis of prospective cohort, case-control, and cross-sectional studies suggested that higher levels of dietary tea consumption can reduce the risk of stroke. ${ }^{53}$ However, another meta-analysis concluded that black tea consumption had no significant effect on total, LDL, or HDL cholesterol, ${ }^{59}$ suggesting that the effects of flavan-3-ols on lipid parameters are likely small. A meta-analysis of randomized, controlled trials reported that green tea flavan-3-ols with or without caffeine significantly reduced fasting glucose but produced no effects on other diabetes-related outcomes. ${ }^{78}$ Another meta-analysis of randomized, controlled trials indicated that higher doses of epicatechin were related to improvements in biomarkers for cardiovascular disease risk factors such as FMD and systolic and diastolic blood pressure. ${ }^{62}$ On balance, the positive effects of tea polyphenols on cardiovascular disease relate to overall improvements in vascular function $^{79}$ and blood pressure. ${ }^{80}$ It is important to consider that during the production of black tea most of the catechins are oxidized to condensed flavonoids such as theaflavins and thearubigins. These have complex chemical structures, and their biological activities and participation in the health benefits of tea are still being defined. ${ }^{80,81}$

4.1.2. Anthocyanins. Anthocyanins are flavonoids that are mainly found in red and blue fruits and vegetables, in particular blueberries, raspberries, red grapes, and cherries. After being consumed, anthocyanins, like other polyphenols, are 
metabolized by the host and the microbiome to form active metabolites that have anti-inflammatory properties and produce positive vascular effects. ${ }^{82}$ Prospective cohort studies have demonstrated that high intake of anthocyanins is associated with a reduced risk of myocardial infarction in men and women and produce other positive effects on cardiovascular risk factors. ${ }^{82-84}$ Two meta-analyses of randomized, controlled trials that evaluated consumption of different sources of anthocyanins (e.g. juices, whole berries, extracts) was associated with a significant reduction in LDL cholesterol, systolic blood pressure, fasting glucose, HbA1c, and body mass index. ${ }^{64,85} \mathrm{~A}$ recently published meta-analysis of randomized, controlled trials that utilized a purified anthocyanin supplement reported significant benefits in glycemic control and lipid parameters in individuals with pre-diabetes or early untreated diabetes. ${ }^{86} \mathrm{~A}$ meta-analysis of randomized, controlled trials that evaluated consumption of anthocyanin-rich foods or supplements reported improvements in vascular functioning, such as FMD and arterial stiffness, ${ }^{87}$ while another meta-analysis reported significant reductions in triglycerides and total and LDL cholesterol. ${ }^{88}$

4.1.3. Stilbenes. Resveratrol is a stilbene and is principally found in grapes, red wine, and berries. ${ }^{89}$ Resveratrol has been widely studied, and several mechanisms of action have been proposed, including inhibition of cytokine release and modulation of NO synthesis ${ }^{89}$ resulting in anti-inflammatory and antioxidant actions. A relevant mechanism of action for resveratrol is the activation of sirtuins, ${ }^{90}$ which have been associated with a delay of aging. ${ }^{91}$ A meta-analysis of randomized, controlled trials reported that resveratrol supplementation significantly reduced fasting glucose, total cholesterol, C-reactive protein (CRP), and systolic and diastolic blood pressure; these effects were particularly pronounced in those with pre-existing NCDs (e.g. type 2 diabetes, cardiovascular disease).$^{92}$ However, two older meta-analyses reported that resveratrol had no effect on CRP, lipid profiles, and several other cardiovascular risk factors. ${ }^{93,94}$ Other meta-analyses of randomized, controlled trials reported that resveratrol had beneficial effects on nonalcoholic fatty liver disease by modulating lipid parameters ${ }^{95}$ and on improved glucose control and insulin sensitivity in individuals with cardiometabolic conditions. ${ }^{96}$ Furthermore, resveratrol has been shown to improve peripheral blood flow, as assessed by FMD, in overweight or obese individuals following administration of single doses and extended periods of supplementation. ${ }^{97,98}$

4.1.4. Flavonols. Quercetin is a flavonol that has been shown to improve endothelial functioning in a randomized, controlled trial by modulating circulating concentrations of vasoactive NO products and endothelin-1. ${ }^{99} \mathrm{~A}$ randomized, controlled trial that evaluated a flavonoid-rich apple reported increases in plasma quercetin concentrations and significantly improved endothelial function in individuals at risk for cardiovascular disease. ${ }^{58}$ Another controlled trial conducted with subjects at risk for cardiovascular disease reported that quercetin supplementation significantly reduced systolic blood pressure and LDL cholesterol. ${ }^{100}$ A meta-analysis of random- ized, controlled trials conducted with quercetin supplements observed a significant reduction in systolic and diastolic blood pressure, particularly at doses $\geq 500 \mathrm{mg}$ day $^{-1} \cdot{ }^{63}$ However, some individual studies reported no significant effect of isolated or pure quercetin at lower doses on blood pressure or endothelial function or on general cardiometabolic health. ${ }^{101,102}$

\subsection{Effects of polyphenols on cognition}

In addition to the effects of polyphenols on cardiometabolic health, polyphenols are also thought to have beneficial effects on cognitive functioning. A prospective study of adults in midlife found a positive association between total polyphenol intake and cognitive factors (i.e. language and verbal memory) assessed over 13 years. ${ }^{103}$ In longitudinal studies, regular dietary chocolate consumption has also been shown to reduce the risk of experiencing cognitive decline. ${ }^{104,105}$ In addition, in a meta-analysis of 17 independent observational studies, tea consumption was found to have an inverse linear relationship with the incidence of cognitive disorders. ${ }^{6}$ Other observational studies have also shown that green and black tea consumption is related to a lower risk of experiencing cognitive impairment. ${ }^{106,107}$ Tea consumption has further been found to independently reduce the risk for experiencing depression ${ }^{108}$ and may have a protective effect against Parkinson's disease. ${ }^{109}$

There is clear evidence to suggest that polyphenol-rich products and single polyphenols consistently increase cerebral blood flow (CBF) or modulate brain activity after single doses ${ }^{110-117}$ and longer periods of supplementation. ${ }^{118-124}$ However, while most of these CBF studies included an assessment of cognitive task performance or mood, only a very small minority ${ }^{117,123,124}$ reported any significant improvements in these domains in comparison with placebo.

In long-term intervention studies, the strongest evidence of flavan-3-ols producing benefits in cognition comes from two studies with similar methodologies conducted in healthy elderly participants ${ }^{125}$ and sufferers of age-related cognitive impairment ( $n=90$ per study). ${ }^{126}$ Subjects consumed drinks with low (control), medium (520 mg), or high (990 mg) flavan3-ol content over an 8-week treatment period. Both studies reported that the high-flavan-3-ol drink reduced insulin resistance, blood pressure, and lipid peroxidation and improved attention and executive function. Similar results were observed in those who consumed the medium-flavan-3-ol content drinks for the executive functioning task, but no improvement on the attention task was observed. ${ }^{125,126}$ These observed benefits of flavan-3-ols are further supported by a study that enrolled 40 elderly participants who were supplemented over 12 weeks with $494 \mathrm{mg}$ of flavan-3-ols showing improved scores on a composite cognitive measure derived from a battery of tasks. ${ }^{127}$ Furthermore, blood levels of brain-derived neurotrophic factor, a protein involved in the survival, growth, and differentiation of new neurons and synapses, were also increased by flavan-3-ol supplementation. ${ }^{127}$ 


\section{Polyphenols and the potential interaction with other bioactive food components}

When interpreting the results of many of the clinical studies conducted to assess the influence of polyphenols on a health parameter, consideration of whether the interventions were based on consumption of whole foods, purified extracts, or isolated compounds must be made. ${ }^{128}$ Indeed, the effects of potentially complex interventions with multiple bioactive components, as in the case of whole foods, have to be carefully interpreted. Advantageous complementary, additive, or synergistic interactions between polyphenols and/or other classes of phytochemicals, as well as negative or neutralizing effects of different compounds, are situations that need attention. In this direction, there is a growing need for properly designed in vitro, animal, and human intervention studies considering the potentiality of such interactions.

An example of the importance of the interactions between polyphenols and other substances is provided by the study of cocoa products in vascular health. Primarily, the vascular effects of cocoa are attributed to flavan-3-ols, but the presence of comparatively low amounts of caffeine can also produce vascular effects. As a result, intervention studies have typically matched the methylxanthine (i.e. caffeine) content for the test and control treatments. ${ }^{129}$ While it seems appropriate to match caffeine content to separate out the effects of the flavan3-ols, additional considerations should be taken because of the potential for interactions between flavan-3-ols and caffeine. A recent randomized, controlled trial reported that the vascular effects of cocoa flavanols, including FMD, were enhanced by the co-ingestion of methylxanthines, which were associated with an increase in plasma concentrations of epicatechin-related metabolites. ${ }^{129}$ Another study in this direction shows that the bioavailability of epigallocatechin-3-gallate (ECGC) is increased when consumed with caffeine. ${ }^{130}$ The authors suggest caffeine suppresses the conjugation of ECGC with increases observed in plasma concentrations, but these interactions may extend beyond increasing bioavailability. It has been suggested that the thermogenic effects of ECGC present in green tea extracts are driven at least in part by tea flavanols, caffeine, and their combined effects on norepinephrine. ${ }^{131}$ The increases in thermogenesis are likely due to a combined effect in which the flavanols inhibit catechol $O$-methyltransferase, prolonging the duration of norepinephrine retention in the synaptic cleft, while caffeine inhibits the action of phosphodiesterase in cyclic AMP in the cells of adipose tissue, both resulting in a prolonged effect of norepinephrine on thermogenesis. ${ }^{131}$ In another recent randomized, controlled, cross-over study by Boolani et al., the addition of caffeine to cocoa may have acted to improve accuracy and reduce omission errors on the Bakan primary task. ${ }^{132}$ However, due to the inclusion of a control group that did not receive caffeine, it is difficult to differentiate the effects of the caffeine from the cocoa polyphenols. There is also a large body of research that attributes the cognitive effects of tea and coffee to caffeine without any consideration for any contribution from polyphenols. ${ }^{133,134}$ However, if caffeine were the only active component, tea and coffee with the same caffeine content would be expected to have similar effects. Evidence from intervention trials directly comparing single doses of tea and coffee also shows that their effects differ in terms of physiological responses ${ }^{135}$ and psychological functioning. ${ }^{136,137}$ Similarly, tea, when compared directly with its caffeine content, has different effects than caffeine alone on stress responses, mood, and overall somatic metabolism. ${ }^{138,139}$ As an example of the physiological effects, one study ${ }^{131}$ found that green tea increased fat oxidation and decreased carbohydrate oxidation as the percent of total energy expenditure over 24 hours in a respiratory chamber in comparison to its caffeine content alone. Likewise, coffee, when compared directly to its caffeine content alone, has different effects on blood pressure, ${ }^{140}$ physical endurance performance, ${ }^{141}$ resistance exercise ${ }^{142}$ and plasma levels of the neurotransmitter epinephrine. ${ }^{141}$

Polyphenols often occur alongside terpenes in plant-based products. For instance, standardized Ginkgo biloba extracts contain $\sim 24 \%$ flavonoids and $6 \%$ diterpenes and their derivatives, and both of these components might have separate, potentially additive, effects on brain function. ${ }^{143}$ However, such potential additive effects have never been experimentally confirmed. Indeed, terpenoids are thought to be the active component of many herbal extracts that exert effects on central nervous system function, including valerian root, which contains iridoids and sesquiterpenes, Panax ginseng, which is rich in triterpenes, and the Mentha and Salvia (sage) genera, which predominantly express volatile monoterpenes. ${ }^{144}$ All of these structural groups of terpenes exert markedly different mechanistic effects relevant to brain function, and they also co-exist in extracts alongside significant quantities of polyphenols. To give an example, the cognitive and mood effects of sage essential oils containing only monoterpenes are similar to those seen with sage extracts combining terpenes with phenolics. ${ }^{144}$ Conversely, an aqueous extract of spearmint containing polyphenols but none of the plant's substantial monoterpene content improved cognitive function in older adults. ${ }^{145}$ Similarly, lemon balm, which has been shown to produce acute anxiolytic and cognitive effects, contains flavonoids as well as monoterpenoid aldehydes and glycosides. ${ }^{146}$ Therefore, it appears that different classes of terpenes have distinct mechanisms of action that produce varying effects.

The cardiovascular benefits that have been observed with polyphenols may also be due to the cooperative effects with other bioactive compounds, such as nitrates and fibers. Consuming polyphenol-rich fruits with a Western style meal can attenuate the resulting inflammatory processes associated with high-energy dietary patterns. ${ }^{147}$ In one study, consuming either green tea or fruit-based polyphenols and fiber had positive effects on post-prandial glucose and insulin when consumed together with starchy food. ${ }^{148}$ In a recent study, pomegranate juice consumption modulated the post-prandial glycemic response; however, the same polyphenols present in 
pomegranates but taken as a supplement did not. The authors of the work consider that this was possibly due to poor mixing of the polyphenol punicalagin in extract form in the stomach and intestine. ${ }^{149}$ Polyphenols have also been shown to protect readily oxidized nutrients, in particular, lipids such as polyunsaturated fatty acids, which can enhance their beneficial effects. ${ }^{150}$ Flavonoids (e.g. quercetin, (-)-epicatechin, (-)-epigallocatechin gallate) can also protect $\alpha$-tocopherol from oxidation during digestion, which increases $\alpha$-tocopherol concentrations in plasma and liver. ${ }^{151,152}$ Two separate studies from the same group evaluating the effect of consuming flavonoidrich apples with nitrate-rich spinach on NO status reported conflicting results. ${ }^{75,153}$ One study reported that the combination of these two foods augmented NO status, while not showing any impact on cognitive function. ${ }^{153}$ The second study reported a significant interaction between higher NO status (which was independently but not synergistically related to the consumption of flavonoid-rich apples with nitrate-rich spinach) and endothelial dysfunction, as well as increased blood pressure. ${ }^{75}$ Although this group later demonstrated that quercetin had no effects on blood pressure or endothelial function, ${ }^{101}$ a more recent study found that whole apples rich in flavonoids (including the skin) significantly improved endothelial function compared with low-flavonoid apples (without the skin). ${ }^{58}$ Notably, plasma quercetin levels were increased following consumption of the flavonoid-rich apples. The effects of quercetin on endothelial function are thought to be due, in part, to their effects on NO, possibly by stimulating endothelial NO synthase activity. This study, however, was not able to detect small changes in nitrite and nitrate concentrations ${ }^{58}$ which may relate to technical issues associated with measuring nitrite as a marker of NO production in frozen samples rather than fresh plasma. ${ }^{154}$

\section{Conclusions}

Consuming polyphenol-rich foods has been associated with a range of health benefits, including optimizing cardiometabolic health and to a lesser extent positively impacting brain functioning in humans. However, the actual mechanisms for these effects have not been fully established. The complex relationships between polyphenols and the gut microbiome, as well as the interaction between the microbiome and health outcomes, should be considered as relevant participants in these health effects that warrant further research. Furthermore, the possibility of interactions with other bioactives present in foods or that can be administered as supplements, (e.g. fiber, terpenoids, and alkaloids) deserves consideration when designing future studies.

\section{Conflicts of interest}

The authors received an honorarium from Pfizer for attending the workshop that resulted in preparation of this manuscript.
CF consults for Nestle, Nuskin, and Pfizer. DK received grants from Evolva, Frutarom, Mibelle, Nexira, and PepsiCo outside the submitted work. FTB received grants from Hero España, Interquim and Nestlé outside the submitted work.

\section{Acknowledgements}

Medical writing support was provided by Dennis Stancavish of Peloton Advantage, LLC, and was funded by Pfizer.

\section{References}

1 World Health Organization, World Health Statistics 2016 Monitoring Health for the SDGs, Sustainable Development Goals, World Health Organization, Geneva, Switzerland, 2016.

2 World Health Organization, Diet, Nutrition and the Prevention of Chronic Diseases, World Health Organization, Geneva, Switzerland, 2003/05/29th edn, 2003.

3 L. G. Saldanha, Summary of comments received in response to the Federal Register notice defining bioactive food components, https://ods.od.nih.gov/pubs/bioactivefoodcomponents/Summary20of20comments20-20on20defining20bioactive20food20components.pdf, accessed November 9, 2017.

4 P. C. Hollman, A. Geelen and D. Kromhout, Dietary flavonol intake may lower stroke risk in men and women, J. Nutr., 2010, 140, 600-604.

5 L. Hooper, P. A. Kroon, E. B. Rimm, J. S. Cohn, I. Harvey, K. A. Le Cornu, J. J. Ryder, W. L. Hall and A. Cassidy, Flavonoids, flavonoid-rich foods, and cardiovascular risk: a meta-analysis of randomized controlled trials, Am. J. Clin. Nutr., 2008, 88, 38-50.

6 X. Liu, X. Du, G. Han and W. Gao, Association between tea consumption and risk of cognitive disorders: A doseresponse meta-analysis of observational studies, Oncotarget, 2017, 8, 43306-43321.

7 L. Shen, L. G. Song, H. Ma, C. N. Jin, J. A. Wang and M. X. Xiang, Tea consumption and risk of stroke: a doseresponse meta-analysis of prospective studies, J. Zhejiang Univ., Sci., B, 2012, 13, 652-662.

8 J. R. Lupton, S. A. Atkinson, N. Chang, C. G. Fraga, J. Levy, M. Messina, D. P. Richardson, B. van Ommen, Y. Yang, J. C. Griffiths and J. Hathcock, Exploring the benefits and challenges of establishing a DRI-like process for bioactives, Eur. J. Nutr., 2014, 53(Suppl 1), 1-9.

9 M. Mazid, T. A. Khan and F. Mohammad, Role of secondary metabolites in defense mechanisms of plants, Biol. Med., 2011, 3, 232-249.

10 L. Marin, E. M. Miguelez, C. J. Villar and F. Lombo, Bioavailability of dietary polyphenols and gut microbiota metabolism: antimicrobial properties, BioMed. Res. Int., 2015, 2015, 905215. 
11 D. O. Kennedy, Polyphenols and the human brain: plant "secondary metabolite" ecologic roles and endogenous signaling functions drive benefits, Adv. Nutr., 2014, 5, 515-533.

12 C. Manach, G. Williamson, C. Morand, A. Scalbert and C. Remesy, Bioavailability and bioefficacy of polyphenols in humans. I. Review of 97 bioavailability studies, Am. J. Clin. Nutr., 2005, 81, 230s-242s.

13 S. K. Bohn, N. C. Ward, J. M. Hodgson and K. D. Croft, Effects of tea and coffee on cardiovascular disease risk, Food Funct., 2012, 3, 575-591.

14 M. P. Corcoran, D. L. McKay and J. B. Blumberg, Flavonoid basics: chemistry, sources, mechanisms of action, and safety, J. Nutr. Gerontol. Geriatr., 2012, 31, 176-189.

15 Phenol-Explorer 3.6. Database on polyphenol content in foods, http://phenol-explorer.eu/compounds/classification, accessed April 4, 2018.

16 A. P. Butiuk, M. A. Martos, O. Adachi and R. A. Hours, Study of the chlorogenic acid content of in yerba mate (Ilex paraguariensis St. Hil.): Effect of plant fraction, processing step and harvesting season, J. Appl. Res. Med. Aromat. Plants, 2016, 3, 27-33.

17 A. Crozier, D. Del Rio and M. N. Clifford, Bioavailability of dietary flavonoids and phenolic compounds, Mol. Aspects Med., 2010, 31, 446-467.

18 M. V. Selma, J. C. Espin and F. A. Tomas-Barberan, Interaction between phenolics and gut microbiota: role in human health, J. Agric. Food Chem., 2009, 57, 6485-6501.

19 V. Fogliano, M. L. Corollaro, P. Vitaglione, A. Napolitano, R. Ferracane, F. Travaglia, M. Arlorio, A. Costabile, A. Klinder and G. Gibson, In vitro bioaccessibility and gut biotransformation of polyphenols present in the water-insoluble cocoa fraction, Mol. Nutr. Food Res., 2011, 55(Suppl 1), S44-S55.

20 P. I. Oteiza, C. G. Fraga, D. A. Mills and D. H. Taft, Flavonoids and the gastrointestinal tract: Local and systemic effects, Mol. Aspects Med., 2018, 61, 41-49.

21 F. A. Tomas-Barberan, M. V. Selma and J. C. Espin, Interactions of gut microbiota with dietary polyphenols and consequences to human health, Curr. Opin. Clin. Nutr. Metab. Care, 2016, 19, 471-476.

22 A. Gonzalez-Sarrias, J. C. Espin and F. A. Tomas-Barberan, Non-extractable polyphenols produce gut microbiota metabolites that persist in circulation and show antiinflammatory and free radical-scavenging effects, Trends Food Sci. Technol., 2017, 69, 281-288.

23 M. Arumugam, J. Raes, E. Pelletier, D. Le Paslier, T. Yamada, D. R. Mende, G. R. Fernandes, J. Tap, T. Bruls, J. M. Batto, M. Bertalan, N. Borruel, F. Casellas, L. Fernandez, L. Gautier, T. Hansen, M. Hattori, T. Hayashi, M. Kleerebezem, K. Kurokawa, M. Leclerc, F. Levenez, C. Manichanh, H. B. Nielsen, T. Nielsen, N. Pons, J. Poulain, J. Qin, T. Sicheritz-Ponten, S. Tims, D. Torrents, E. Ugarte, E. G. Zoetendal, J. Wang, F. Guarner, O. Pedersen, W. M. de Vos, S. Brunak, J. Dore,
M. Antolin, F. Artiguenave, H. M. Blottiere, M. Almeida, C. Brechot, C. Cara, C. Chervaux, A. Cultrone, C. Delorme, G. Denariaz, R. Dervyn, K. U. Foerstner, C. Friss, M. van de Guchte, E. Guedon, F. Haimet, W. Huber, J. van Hylckama-Vlieg, A. Jamet, C. Juste, G. Kaci, J. Knol, O. Lakhdari, S. Layec, K. Le Roux, E. Maguin, A. Merieux, R. Melo Minardi, C. M'Rini, J. Muller, R. Oozeer, J. Parkhill, P. Renault, M. Rescigno, N. Sanchez, S. Sunagawa, A. Torrejon, K. Turner, G. Vandemeulebrouck, E. Varela, Y. Winogradsky, G. Zeller, J. Weissenbach, S. D. Ehrlich and P. Bork, Enterotypes of the human gut microbiome, Nature, 2011, 473, 174-180.

24 J. C. Espin, A. Gonzalez-Sarrias and F. A. Tomas-Barberan, The gut microbiota: A key factor in the therapeutic effects of (poly)phenols, Biochem. Pharmacol., 2017, 139, 82-93.

25 M. V. Selma, D. Beltran, M. C. Luna, M. Romo-Vaquero, R. Garcia-Villalba, A. Mira, J. C. Espin and F. A. TomasBarberan, Isolation of human intestinal bacteria capable of producing the bioactive metabolite isourolithin A from ellagic acid, Front. Microbiol., 2017, 8, 1521.

26 M. V. Selma, D. Beltran, R. Garcia-Villalba, J. C. Espin and F. A. Tomas-Barberan, Description of urolithin production capacity from ellagic acid of two human intestinal Gordonibacter species, Food Funct., 2014, 5, 1779-1784.

27 F. A. Tomas-Barberan, R. Garcia-Villalba, A. GonzalezSarrias, M. V. Selma and J. C. Espin, Ellagic acid metabolism by human gut microbiota: consistent observation of three urolithin phenotypes in intervention trials, independent of food source, age, and health status, J. Agric. Food Chem., 2014, 62, 6535-6538.

28 B. Cerda, F. A. Tomas-Barberan and J. C. Espin, Metabolism of antioxidant and chemopreventive ellagitannins from strawberries, raspberries, walnuts, and oakaged wine in humans: identification of biomarkers and individual variability, J. Agric. Food Chem., 2005, 53, 227235.

29 C. L. Frankenfeld, Cardiometabolic risk and gut microbial phytoestrogen metabolite phenotypes, Mol. Nutr. Food Res., 2017, 61(1), DOI: 10.1002/mnfr.201500900.

30 T. R. Sampson, J. W. Debelius, T. Thron, S. Janssen, G. G. Shastri, Z. E. Ilhan, C. Challis, C. E. Schretter, S. Rocha, V. Gradinaru, M. F. Chesselet, A. Keshavarzian, K. M. Shannon, R. Krajmalnik-Brown, P. WittungStafshede, R. Knight and S. K. Mazmanian, Gut microbiota regulate motor deficits and neuroinflammation in a model of Parkinson's disease, Cell, 2016, 167, 1469-1480.

31 V. K. Ridaura, J. J. Faith, F. E. Rey, J. Cheng, A. E. Duncan, A. L. Kau, N. W. Griffin, V. Lombard, B. Henrissat, J. R. Bain, M. J. Muehlbauer, O. Ilkayeva, C. F. Semenkovich, K. Funai, D. K. Hayashi, B. J. Lyle, M. C. Martini, L. K. Ursell, J. C. Clemente, W. Van Treuren, W. A. Walters, R. Knight, C. B. Newgard, A. C. Heath and J. I. Gordon, Gut microbiota from twins discordant for obesity modulate metabolism in mice, Science, 2013, 341, 1241214. 
32 S. Masumoto, A. Terao, Y. Yamamoto, T. Mukai, T. Miura and T. Shoji, Non-absorbable apple procyanidins prevent obesity associated with gut microbial and metabolomic changes, Sci. Rep., 2016, 6, 31208.

33 A. Duda-Chodak, T. Tarko, P. Satora and P. Sroka, Interaction of dietary compounds, especially polyphenols, with the intestinal microbiota: a review, Eur. J. Nutr., 2015, 54, 325-341.

34 D. Gwiazdowska, K. Jus, J. Jasnowska-Malecka and K. Kluczynska, The impact of polyphenols on Bifidobacterium growth, Acta Biochim. Pol., 2015, 62, 895-901.

35 J. S. Jin, M. Touyama, T. Hisada and Y. Benno, Effects of green tea consumption on human fecal microbiota with special reference to Bifidobacterium species, Microbiol. Immunol., 2012, 56, 729-739.

36 A. Koutsos, M. Lima, L. Conterno, M. Gasperotti, M. Bianchi, F. Fava, U. Vrhovsek, J. A. Lovegrove and K. M. Tuohy, Effects of commercial apple varieties on human gut microbiota composition and metabolic output using an in vitro colonic model, Nutrients, 2017, 9(6), pii: E533.

37 S. Vendrame, S. Guglielmetti, P. Riso, S. Arioli, D. KlimisZacas and M. Porrini, Six-week consumption of a wild blueberry powder drink increases bifidobacteria in the human gut, J. Agric. Food Chem., 2011, 59, 1281512820.

38 S. Raimondi, A. Anighoro, A. Quartieri, A. Amaretti, F. A. Tomas-Barberan, G. Rastelli and M. Rossi, Role of bifidobacteria in the hydrolysis of chlorogenic acid, MicrobiologyOpen, 2015, 4, 41-52.

39 F. Tomas-Barberan, R. Garcia-Villalba, A. Quartieri, S. Raimondi, A. Amaretti, A. Leonardi and M. Rossi, In vitro transformation of chlorogenic acid by human gut microbiota, Mol. Nutr. Food Res., 2014, 58, 11221131.

40 C. G. Fraga, M. Galleano, S. V. Verstraeten and P. I. Oteiza, Basic biochemical mechanisms behind the health benefits of polyphenols, Mol. Aspects Med., 2010, 31, 435445.

41 M. Galleano, S. V. Verstraeten, P. I. Oteiza and C. G. Fraga, Antioxidant actions of flavonoids: thermodynamic and kinetic analysis, Arch. Biochem. Biophys., 2010, 501, 23-30.

42 C. G. Fraga, Plant polyphenols: how to translate their in vitro antioxidant actions to in vivo conditions, IUBMB Life, 2007, 59, 308-315.

43 C. G. Fraga and P. I. Oteiza, Dietary flavonoids: Role of (-)-epicatechin and related procyanidins in cell signaling, Free Radicals Biol. Med., 2011, 51, 813-823.

44 H. J. Forman, K. J. Davies and F. Ursini, How do nutritional antioxidants really work: nucleophilic tone and para-hormesis versus free radical scavenging in vivo, Free Radicals Biol. Med., 2014, 66, 24-35.

45 H. S. Kim, M. J. Quon and J. A. Kim, New insights into the mechanisms of polyphenols beyond antioxidant properties; lessons from the green tea polyphenol, epigallocatechin 3-gallate, Redox Biol., 2014, 2, 187-195.
46 C. G. Fraga, P. I. Oteiza and M. Galleano, Plant bioactives and redox signaling: (-)-Epicatechin as a paradigm, Mol. Aspects Med., 2018, 61, 31-40.

47 J. R. Ehrenkranz, N. G. Lewis, C. R. Kahn and J. Roth, Phlorizin: a review, Diabetes/Metab. Res. Rev., 2005, 21, 3138.

48 E. Cremonini, A. Bettaieb, F. G. Haj, C. G. Fraga and P. I. Oteiza, (-)-Epicatechin improves insulin sensitivity in high fat diet-fed mice, Arch. Biochem. Biophys., 2016, 599, 13-21.

49 A. Bettaieb, E. Cremonini, H. Kang, J. Kang, F. G. Haj and P. I. Oteiza, Anti-inflammatory actions of (-)-epicatechin in the adipose tissue of obese mice, Int. J. Biochem. Cell Biol., 2016, 81, 383-392.

50 M. A. Vazquez-Prieto, A. Bettaieb, F. G. Haj, C. G. Fraga and P. I. Oteiza, (-)-Epicatechin prevents TNFalphainduced activation of signaling cascades involved in inflammation and insulin sensitivity in 3 T3-L1 adipocytes, Arch. Biochem. Biophys., 2012, 527, 113-118.

51 A. Buitrago-Lopez, J. Sanderson, L. Johnson, S. Warnakula, A. Wood, E. Di Angelantonio and O. H. Franco, Chocolate consumption and cardiometabolic disorders: systematic review and meta-analysis, $\mathrm{Br}$. Med. J., 2011, 343, d4488.

52 G. E. Crichton, M. F. Elias, P. J. Dearborn and M. A. Robbins, Habitual chocolate intake and type 2 diabetes mellitus in the Maine-Syracuse Longitudinal Study: (1975-2010): prospective observations, Appetite, 2017, 108, 263-269.

53 L. Arab, W. Liu and D. Elashoff, Green and black tea consumption and risk of stroke: a meta-analysis, Stroke, 2009, 40, 1786-1792.

54 A. Afshin, R. Micha, S. Khatibzadeh and D. Mozaffarian, Consumption of nuts and legumes and risk of incident ischemic heart disease, stroke, and diabetes: a systematic review and meta-analysis, Am. J. Clin. Nutr., 2014, 100, 278-288.

55 C. S. Kwok, S. M. Boekholdt, M. A. Lentjes, Y. K. Loke, R. N. Luben, J. K. Yeong, N. J. Wareham, P. K. Myint and K. T. Khaw, Habitual chocolate consumption and risk of cardiovascular disease among healthy men and women, Heart, 2015, 101, 1279-1287.

56 J. A. Greenberg, Chocolate intake and diabetes risk, Clin. Nutr., 2015, 34, 129-133.

57 S. C. Larsson, A. Akesson, B. Gigante and A. Wolk, Chocolate consumption and risk of myocardial infarction: a prospective study and meta-analysis, Heart, 2016, 102, 1017-1022.

58 N. P. Bondonno, C. P. Bondonno, L. C. Blekkenhorst, M. J. Considine, G. Maghzal, R. Stocker, R. J. Woodman, N. C. Ward, J. M. Hodgson and K. D. Croft, Flavonoid-rich apple improves endothelial function in individuals at risk for cardiovascular disease: a randomized controlled clinical trial, Mol. Nutr. Food Res., 2018, 62(3), DOI: 10.1002/ mnfr.201700674.

59 D. Wang, C. Chen, Y. Wang, J. Liu and R. Lin, Effect of black tea consumption on blood cholesterol: a meta-ana- 
lysis of 15 randomized controlled trials, PLoS One, 2014, 9, e107711.

60 X. Lin, I. Zhang, A. Li, J. E. Manson, H. D. Sesso, L. Wang and S. Liu, Cocoa flavanol intake and biomarkers for cardiometabolic health: a systematic review and meta-analysis of randomized controlled trials, J. Nutr., 2016, 146, 2325-2333.

61 K. Ried, T. Sullivan, P. Fakler, O. R. Frank and N. P. Stocks, Does chocolate reduce blood pressure? A meta-analysis, BMC Med., 2010, 8, 39.

62 L. Hooper, C. Kay, A. Abdelhamid, P. A. Kroon, J. S. Cohn, E. B. Rimm and A. Cassidy, Effects of chocolate, cocoa, and flavan-3-ols on cardiovascular health: a systematic review and meta-analysis of randomized trials, Am. J. Clin. Nutr., 2012, 95, 740-751.

63 M. C. Serban, A. Sahebkar, A. Zanchetti, D. P. Mikhailidis, G. Howard, D. Antal, F. Andrica, A. Ahmed, W. S. Aronow, P. Muntner, G. Y. Lip, I. Graham, N. Wong, J. Rysz and M. Banach, Effects of quercetin on blood pressure: a systematic review and meta-analysis of randomized controlled trials, J. Am. Heart Assoc., 2016, 5(7), pii: e002713.

64 H. Huang, G. Chen, D. Liao, Y. Zhu and X. Xue, Effects of berries consumption on cardiovascular risk factors: a meta-analysis with trial sequential analysis of randomized controlled trials, Sci. Rep., 2016, 6, 23625.

65 A. Tresserra-Rimbau, E. B. Rimm, A. Medina-Remon, M. A. Martinez-Gonzalez, M. C. Lopez-Sabater, M. I. Covas, D. Corella, J. Salas-Salvado, E. Gomez-Gracia, J. Lapetra, F. Aros, M. Fiol, E. Ros, L. Serra-Majem, X. Pinto, M. A. Munoz, A. Gea, V. Ruiz-Gutierrez, R. Estruch and R. M. Lamuela-Raventos, Polyphenol intake and mortality risk: a re-analysis of the PREDIMED trial, BMC Med., 2014, 12, 77.

66 A. Tresserra-Rimbau, E. B. Rimm, A. Medina-Remon, M. A. Martinez-Gonzalez, R. de la Torre, D. Corella, J. Salas-Salvado, E. Gomez-Gracia, J. Lapetra, F. Aros, M. Fiol, E. Ros, L. Serra-Majem, X. Pinto, G. T. Saez, J. Basora, J. V. Sorli, J. A. Martinez, E. Vinyoles, V. RuizGutierrez, R. Estruch and R. M. Lamuela-Raventos, Inverse association between habitual polyphenol intake and incidence of cardiovascular events in the PREDIMED study, Nutr., Metab. Cardiovasc. Dis., 2014, 24, 639-647.

67 M. A. Kelm, J. C. Johnson, R. J. Robbins, J. F. Hammerstone and H. H. Schmitz, High-performance liquid chromatography separation and purification of cacao (Theobroma cacao L.) procyanidins according to degree of polymerization using a diol stationary phase, J. Agric. Food Chem., 2006, 54, 1571-1576.

68 S. A. Lazarus, J. F. Hammerstone, G. E. Adamson and H. H. Schmitz, High-performance liquid chromatography/ mass spectrometry analysis of proanthocyanidins in food and beverages, Methods Enzymol., 2001, 335, 46-57.

69 L. Gu, M. A. Kelm, J. F. Hammerstone, G. Beecher, J. Holden, D. Haytowitz and R. L. Prior, Screening of foods containing proanthocyanidins and their structural characterization using LC-MS/MS and thiolytic degradation, J. Agric. Food Chem., 2003, 51, 7513-7521.

70 C. G. Fraga, L. Actis-Goretta, J. I. Ottaviani, F. Carrasquedo, S. B. Lotito, S. Lazarus, H. H. Schmitz and C. L. Keen, Regular consumption of a flavanol-rich chocolate can improve oxidant stress in young soccer players, Clin. Dev. Immunol., 2005, 12, 11-17.

71 G. E. Crichton, M. F. Elias, A. Alkerwi, S. Stranges and W. P. Abhayaratna, Relation of habitual chocolate consumption to arterial stiffness in a community-based sample: preliminary findings, Pulse, 2016, 4, 28-37.

72 M. L. McCullough, J. J. Peterson, R. Patel, P. F. Jacques, R. Shah and J. T. Dwyer, Flavonoid intake and cardiovascular disease mortality in a prospective cohort of US adults, Am. J. Clin. Nutr., 2012, 95, 454-464.

73 C. D. Kay, L. Hooper, P. A. Kroon, E. B. Rimm and A. Cassidy, Relative impact of flavonoid composition, dose and structure on vascular function: a systematic review of randomised controlled trials of flavonoid-rich food products, Mol. Nutr. Food Res., 2012, 56, 1605-1616.

74 M. G. Shrime, S. R. Bauer, A. C. McDonald, N. H. Chowdhury, C. E. Coltart and E. L. Ding, Flavonoidrich cocoa consumption affects multiple cardiovascular risk factors in a meta-analysis of short-term studies, J. Nutr., 2011, 141, 1982-1988.

75 C. P. Bondonno, X. Yang, K. D. Croft, M. J. Considine, N. C. Ward, L. Rich, I. B. Puddey, E. Swinny, A. Mubarak and J. M. Hodgson, Flavonoid-rich apples and nitrate-rich spinach augment nitric oxide status and improve endothelial function in healthy men and women: a randomized controlled trial, Free Radicals Biol. Med., 2012, 52, 95102.

76 H. Schroeter, C. Heiss, J. Balzer, P. Kleinbongard, C. L. Keen, N. K. Hollenberg, H. Sies, C. Kwik-Uribe, H. H. Schmitz and M. Kelm, (-)-Epicatechin mediates beneficial effects of flavanol-rich cocoa on vascular function in humans, Proc. Natl. Acad. Sci. U. S. A., 2006, 103, 10241029.

77 J. I. Dower, J. M. Geleijnse, P. Hollman, S. S. SoedamahMuthu and D. Kromhout, Dietary epicatechin intake and 25-y risk of cardiovascular mortality: the Zutphen Elderly Study, Am. J. Clin. Nutr., 2016, 104, 58-64.

78 X. X. Zheng, Y. L. Xu, S. H. Li, R. Hui, Y. J. Wu and X. H. Huang, Effects of green tea catechins with or without caffeine on glycemic control in adults: a metaanalysis of randomized controlled trials, Am. J. Clin. Nutr., 2013, 97, 750-762.

79 R. T. Ras, P. L. Zock and R. Draijer, Tea consumption enhances endothelial-dependent vasodilation; a metaanalysis, PLoS One, 2011, 6, e16974.

80 J. M. Hodgson, I. B. Puddey, R. J. Woodman, T. P. Mulder, D. Fuchs, K. Scott and K. D. Croft, Effects of black tea on blood pressure: a randomized controlled trial, Arch. Intern. Med., 2012, 172, 186-188.

81 J. M. Hodgson and K. D. Croft, Tea flavonoids and cardiovascular health, Mol. Aspects Med., 2010, 31, 495-502. 
82 A. Cassidy, Berry anthocyanin intake and cardiovascular health, Mol. Aspects Med., 2018, 61, 76-82.

83 A. Cassidy, K. J. Mukamal, L. Liu, M. Franz, A. H. Eliassen and E. B. Rimm, High anthocyanin intake is associated with a reduced risk of myocardial infarction in young and middle-aged women, Circulation, 2013, 127, 188-196.

84 A. Cassidy, M. Bertoia, S. Chiuve, A. Flint, J. Forman and E. B. Rimm, Habitual intake of anthocyanins and flavanones and risk of cardiovascular disease in men, Am. J. Clin. Nutr., 2016, 104, 587-594.

85 L. Yang, W. Ling, Z. Du, Y. Chen, D. Li, S. Deng, Z. Liu and L. Yang, Effects of anthocyanins on cardiometabolic health: A systematic teview and meta-analysis of randomized controlled trials, Adv. Nutr., 2017, 8, 684-693.

86 L. Yang, W. Ling, Y. Yang, Y. Chen, Z. Tian, Z. Du, J. Chen, Y. Xie, Z. Liu and L. Yang, Role of purified anthocyanins in improving cardiometabolic risk factors in Chinese men and women with prediabetes or early untreated diabetes-a randomized controlled trial, Nutrients, 2017, 9(10), pii: E1104.

87 L. Fairlie-Jones, K. Davison, E. Fromentin and A. M. Hill, The effect of anthocyanin-rich foods or extracts on vascular function in adults: A systematic review and meta-analysis of randomised controlled trials, Nutrients, 2017, 9(8), pii: E908.

88 C. Liu, J. Sun, Y. Lu and Y. Bo, Effects of anthocyanin on serum lipids in dyslipidemia patients: A systematic review and meta-analysis, PLoS One, 2016, 11, e0162089.

89 J. Tome-Carneiro, M. Larrosa, A. Gonzalez-Sarrias, F. A. Tomas-Barberan, M. T. Garcia-Conesa and J. C. Espin, Resveratrol and clinical trials: the crossroad from in vitro studies to human evidence, Curr. Pharm. Des., 2013, 19, 6064-6093.

90 M. Gertz, G. T. Nguyen, F. Fischer, B. Suenkel, C. Schlicker, B. Franzel, J. Tomaschewski, F. Aladini, C. Becker, D. Wolters and C. Steegborn, A molecular mechanism for direct sirtuin activation by resveratrol, PLoS One, 2012, 7, e49761.

91 M. S. Bonkowski and D. A. Sinclair, Slowing ageing by design: the rise of $\mathrm{NAD}(+)$ and sirtuin-activating compounds, Nat. Rev. Mol. Cell Biol., 2016, 17, 679-690.

92 X. F. Guo, J. M. Li, J. Tang and D. Li, Effects of resveratrol supplementation on risk factors of non-communicable diseases: A meta-analysis of randomized controlled trials, Crit. Rev. Food Sci. Nutr., 2017, 1-15, DOI: 10.1080/ 10408398.2017.1349076.

93 A. Sahebkar, Effects of resveratrol supplementation on plasma lipids: a systematic review and meta-analysis of randomized controlled trials, Nutr. Rev., 2013, 71, 822-835.

94 A. Sahebkar, C. Serban, S. Ursoniu, N. D. Wong, P. Muntner, I. M. Graham, D. P. Mikhailidis, M. Rizzo, J. Rysz, L. S. Sperling, G. Y. Lip and M. Banach, Lack of efficacy of resveratrol on C-reactive protein and selected cardiovascular risk factors-Results from a systematic review and meta-analysis of randomized controlled trials, Int. J. Cardiol., 2015, 189, 47-55.
95 C. Zhang, W. Yuan, J. Fang, W. Wang, P. He, J. Lei and C. Wang, Efficacy of resveratrol supplementation against non-alcoholic fatty liver disease: A meta-analysis of placebo-controlled clinical trials, PLoS One, 2016, 11, e0161792.

96 K. Liu, R. Zhou, B. Wang and M. T. Mi, Effect of resveratrol on glucose control and insulin sensitivity: a meta-analysis of 11 randomized controlled trials, Am. J. Clin. Nutr., 2014, 99, 1510-1519.

97 R. H. Wong, N. M. Berry, A. M. Coates, J. D. Buckley, J. Bryan, I. Kunz and P. R. Howe, Chronic resveratrol consumption improves brachial flow-mediated dilatation in healthy obese adults, J. Hypertens., 2013, 31, 1819-1827.

98 R. H. Wong, P. R. Howe, J. D. Buckley, A. M. Coates, I. Kunz and N. M. Berry, Acute resveratrol supplementation improves flow-mediated dilatation in overweight/ obese individuals with mildly elevated blood pressure, Nutr., Metab. Cardiovasc. Dis., 2011, 21, 851-856.

99 W. M. Loke, J. M. Hodgson, J. M. Proudfoot, A. J. McKinley, I. B. Puddey and K. D. Croft, Pure dietary flavonoids quercetin and (-)-epicatechin augment nitric oxide products and reduce endothelin-1 acutely in healthy men, Am. J. Clin. Nutr., 2008, 88, 1018-1025.

100 S. Egert, A. Bosy-Westphal, J. Seiberl, C. Kurbitz, U. Settler, S. Plachta-Danielzik, A. E. Wagner, J. Frank, J. Schrezenmeir, G. Rimbach, S. Wolffram and M. J. Muller, Quercetin reduces systolic blood pressure and plasma oxidised low-density lipoprotein concentrations in overweight subjects with a high-cardiovascular disease risk phenotype: a double-blinded, placebocontrolled cross-over study, Br. J. Nutr., 2009, 102, 10651074.

101 N. P. Bondonno, C. P. Bondonno, L. Rich, E. Mas, S. Shinde, N. C. Ward, J. M. Hodgson and K. D. Croft, Acute effects of quercetin-3-O-glucoside on endothelial function and blood pressure: a randomized dose-response study, Am. J. Clin. Nutr., 2016, 104, 97-103.

102 J. I. Dower, J. M. Geleijnse, L. Gijsbers, P. L. Zock, D. Kromhout and P. C. Hollman, Effects of the pure flavonoids epicatechin and quercetin on vascular function and cardiometabolic health: a randomized, double-blind, placebo-controlled, crossover trial, Am. J. Clin. Nutr., 2015, 101, 914-921.

103 E. Kesse-Guyot, L. Fezeu, V. A. Andreeva, M. Touvier, A. Scalbert, S. Hercberg and P. Galan, Total and specific polyphenol intakes in midlife are associated with cognitive function measured 13 years later, J. Nutr., 2012, 142, 76-83.

104 A. Moreira, M. J. Diogenes, A. de Mendonca, N. Lunet and H. Barros, Chocolate consumption is associated with a lower risk of cognitive decline, J. Alzheimer's Dis., 2016, 53, 85-93.

105 G. E. Crichton, M. F. Elias and A. Alkerwi, Chocolate intake is associated with better cognitive function: The Maine-Syracuse Longitudinal Study, Appetite, 2016, 100, 126-132. 
106 S. Kuriyama, A. Hozawa, K. Ohmori, T. Shimazu, T. Matsui, S. Ebihara, S. Awata, R. Nagatomi, H. Arai and I. Tsuji, Green tea consumption and cognitive function: a cross-sectional study from the Tsurugaya Project 1, Am. J. Clin. Nutr., 2006, 83, 355-361.

107 T. P. Ng, L. Feng, M. Niti, E. H. Kua and K. B. Yap, Tea consumption and cognitive impairment and decline in older Chinese adults, Am. J. Clin. Nutr., 2008, 88, 224-231.

108 X. Dong, C. Yang, S. Cao, Y. Gan, H. Sun, Y. Gong, $\mathrm{H}$. Yang, $\mathrm{X}$. Yin and $\mathrm{Z}$. Lu, Tea consumption and the risk of depression: a meta-analysis of observational studies, Aust. N. Z. J. Psychiatry, 2015, 49, 334-345.

109 F. J. Li, H. F. Ji and L. Shen, A meta-analysis of tea drinking and risk of Parkinson's disease, Sci. World J., 2012, 2012, 923464.

110 D. J. Lamport, D. Pal, C. Moutsiana, D. T. Field, C. M. Williams, J. P. Spencer and L. T. Butler, The effect of flavanol-rich cocoa on cerebral perfusion in healthy older adults during conscious resting state: a placebo controlled, crossover, acute trial, Psychopharmacology, 2015, 232, 3227-3234.

111 L. Decroix, C. Tonoli, D. D. Soares, S. Tagougui, E. Heyman and R. Meeusen, Acute cocoa flavanol improves cerebral oxygenation without enhancing executive function at rest or after exercise, Appl. Physiol., Nutr., Metab., 2016, 41, 1225-1232.

112 D. J. Lamport, D. Pal, A. L. Macready, S. Barbosa-Boucas, J. M. Fletcher, C. M. Williams, J. P. Spencer and L. T. Butler, The effects of flavanone-rich citrus juice on cognitive function and cerebral blood flow: an acute, randomised, placebo-controlled cross-over trial in healthy, young adults, Br. J. Nutr., 2016, 116, 2160-2168.

113 D. O. Kennedy, E. L. Wightman, J. L. Reay, G. Lietz, E. J. Okello, A. Wilde and C. F. Haskell, Effects of resveratrol on cerebral blood flow variables and cognitive performance in humans: a double-blind, placebo-controlled, crossover investigation, Am. J. Clin. Nutr., 2010, 91, 15901597.

114 E. L. Wightman, J. L. Reay, C. F. Haskell, G. Williamson, T. P. Dew and D. O. Kennedy, Effects of resveratrol alone or in combination with piperine on cerebral blood flow parameters and cognitive performance in human subjects: a randomised, double-blind, placebo-controlled, cross-over investigation, Br. J. Nutr., 2014, 112, 203-213.

115 E. L. Wightman, C. F. Haskell-Ramsay, J. L. Reay, G. Williamson, T. Dew, W. Zhang and D. O. Kennedy, The effects of chronic trans-resveratrol supplementation on aspects of cognitive function, mood, sleep, health and cerebral blood flow in healthy, young humans, Br. J. Nutr., 2015, 114, 1427-1437.

116 R. H. Wong, R. S. Nealon, A. Scholey and P. R. Howe, Low dose resveratrol improves cerebrovascular function in type 2 diabetes mellitus, Nutr., Metab. Cardiovasc. Dis., 2016, 26, 393-399.

117 R. H. Wong, D. Raederstorff and P. R. Howe, Acute resveratrol consumption improves neurovascular coupling capacity in adults with type 2 diabetes mellitus, Nutrients, 2016, 8(7), pii: E425.

118 H. M. Evans, P. R. Howe and R. H. Wong, Effects of resveratrol on cognitive performance, mood and cerebrovascular function in post-menopausal women; A 14-week randomised placebo-controlled intervention trial, Nutrients, 2017, 9(1), pii: E27.

119 J. L. Bowtell, Z. Aboo-Bakkar, M. E. Conway, A. R. Adlam and J. Fulford, Enhanced task-related brain activation and resting perfusion in healthy older adults after chronic blueberry supplementation, Appl. Physiol., Nutr., Metab., 2017, 42, 773-779.

120 S. T. Francis, K. Head, P. G. Morris and I. A. Macdonald, The effect of flavanol-rich cocoa on the fMRI response to a cognitive task in healthy young people, J. Cardiovasc. Pharmacol., 2006, 47(Suppl 2), S215-S220.

121 F. A. Sorond, L. A. Lipsitz, N. K. Hollenberg and N. D. Fisher, Cerebral blood flow response to flavanol-rich cocoa in healthy elderly humans, Neuropsychiatr. Dis. Treat., 2008, 4, 433-440.

122 T. Köbe, A. V. Witte, A. Schnelle, V. A. Tesky, J. Pantel, J. P. Schuchardt, A. Hahn, J. Bohlken, U. Grittner and A. Floel, Impact of resveratrol on glucose control, hippocampal structure and connectivity, and memory performance in patients with mild cognitive impairment, Front. Neurosci., 2017, 11, 105.

123 A. V. Witte, L. Kerti, D. S. Margulies and A. Floel, Effects of resveratrol on memory performance, hippocampal functional connectivity, and glucose metabolism in healthy older adults, J. Neurosci., 2014, 34, 7862-7870.

124 S. Y. Bookheimer, B. A. Renner, A. Ekstrom, Z. Li, S. M. Henning, J. A. Brown, M. Jones, T. Moody and G. W. Small, Pomegranate juice augments memory and FMRI activity in middle-aged and older adults with mild memory complaints, Evid. Based Complement. Alternat. Med., 2013, 2013, 946298.

125 D. Mastroiacovo, C. Kwik-Uribe, D. Grassi, S. Necozione, A. Raffaele, L. Pistacchio, R. Righetti, R. Bocale, M. C. Lechiara, C. Marini, C. Ferri and G. Desideri, Cocoa flavanol consumption improves cognitive function, blood pressure control, and metabolic profile in elderly subjects: the Cocoa, Cognition, and Aging (CoCoA) Study-a randomized controlled trial, Am. J. Clin. Nutr., 2015, 101, 538-548.

126 G. Desideri, C. Kwik-Uribe, D. Grassi, S. Necozione, L. Ghiadoni, D. Mastroiacovo, A. Raffaele, L. Ferri, R. Bocale, M. C. Lechiara, C. Marini and C. Ferri, Benefits in cognitive function, blood pressure, and insulin resistance through cocoa flavanol consumption in elderly subjects with mild cognitive impairment: the Cocoa, Cognition, and Aging (CoCoA) study, Hypertension, 2012, 60, 794-801.

127 S. Neshatdoust, C. Saunders, S. M. Castle, D. Vauzour, C. Williams, L. Butler, J. A. Lovegrove and J. P. Spencer, High-flavonoid intake induces cognitive improvements linked to changes in serum brain-derived neurotrophic 
factor: Two randomised, controlled trials, Nutr. Healthy Aging, 2016, 4, 81-93.

128 E. Nurk, H. Refsum, C. A. Drevon, G. S. Tell, H. A. Nygaard, K. Engedal and A. D. Smith, Intake of flavonoid-rich wine, tea, and chocolate by elderly men and women is associated with better cognitive test performance, J. Nutr., 2009, 139, 120-127.

129 R. Sansone, J. I. Ottaviani, A. Rodriguez-Mateos, Y. Heinen, D. Noske, J. P. Spencer, A. Crozier, M. W. Merx, M. Kelm, H. Schroeter and C. Heiss, Methylxanthines enhance the effects of cocoa flavanols on cardiovascular function: randomized, double-masked controlled studies, Am. J. Clin. Nutr., 2017, 105, 352-360.

130 K. Nakagawa, K. Nakayama, M. Nakamura, P. Sookwong, T. Tsuduki, H. Niino, F. Kimura and T. Miyazawa, Effects of co-administration of tea epigallocatechin-3-gallate (EGCG) and caffeine on absorption and metabolism of EGCG in humans, Biosci., Biotechnol., Biochem., 2009, 73, 2014-2017.

131 A. G. Dulloo, C. Duret, D. Rohrer, L. Girardier, N. Mensi, M. Fathi, P. Chantre and J. Vandermander, Efficacy of a green tea extract rich in catechin polyphenols and caffeine in increasing $24 \mathrm{~h}$ energy expenditure and fat oxidation in humans, Am. J. Clin. Nutr., 1999, 70, 1040-1045.

132 A. Boolani, J. B. Lindheimer, B. D. Loy, S. Crozier and P. J. O'Connor, Acute effects of brewed cocoa consumption on attention, motivation to perform cognitive work and feelings of anxiety, energy and fatigue: a randomized, placebo-controlled crossover experiment, BMC Nutr., 2017, $3,8$.

133 T. M. McLellan, J. A. Caldwell and H. R. Lieberman, A review of caffeine's effects on cognitive, physical and occupational performance, Neurosci. Biobehav. Rev., 2016, 71, 294-312.

134 L. Arab, F. Khan and H. Lam, Epidemiologic evidence of a relationship between tea, coffee, or caffeine consumption and cognitive decline, Adv. Nutr., 2013, 4, 115-122.

135 P. Quinlan, J. Lane and L. Aspinall, Effects of hot tea, coffee and water ingestion on physiological responses and mood: the role of caffeine, water and beverage type, Psychopharmacology, 1997, 134, 164-173.

136 I. Hindmarch, P. T. Quinlan, K. L. Moore and C. Parkin, The effects of black tea and other beverages on aspects of cognition and psychomotor performance, Psychopharmacology, 1998, 139, 230-238.

137 I. Hindmarch, U. Rigney, N. Stanley, P. Quinlan, J. Rycroft and J. Lane, A naturalistic investigation of the effects of day-long consumption of tea, coffee and water on alertness, sleep onset and sleep quality, Psychopharmacology, 2000, 149, 203-216.

138 A. Steptoe, E. L. Gibson, R. Vuononvirta, E. D. Williams, M. Hamer, J. A. Rycroft, J. D. Erusalimsky and J. Wardle, The effects of tea on psychophysiological stress responsivity and post-stress recovery: a randomised double-blind trial, Psychopharmacology, 2007, 190, 81-89.
139 R. Hursel, W. Viechtbauer, A. G. Dulloo, A. Tremblay, L. Tappy, W. Rumpler and M. S. Westerterp-Plantenga, The effects of catechin rich teas and caffeine on energy expenditure and fat oxidation: a meta-analysis, Obes. Rev., 2011, 12, e573-e581.

140 M. Noordzij, C. S. Uiterwaal, L. R. Arends, F. J. Kok, D. E. Grobbee and J. M. Geleijnse, Blood pressure response to chronic intake of coffee and caffeine: a metaanalysis of randomized controlled trials, J. Hypertens., 2005, 23, 921-928.

141 T. E. Graham, E. Hibbert and P. Sathasivam, Metabolic and exercise endurance effects of coffee and caffeine ingestion, J. Appl. Physiol., 1998, 85, 883-889.

142 D. L. Richardson and N. D. Clarke, Effect of coffee and caffeine ingestion on resistance exercise performance, J. Strength Cond. Res., 2016, 30, 2892-2900.

143 B. S. Oken, D. M. Storzbach and J. A. Kaye, The efficacy of Ginkgo biloba on cognitive function in Alzheimer disease, Arch. Neurol., 1998, 55, 1409-1415.

144 D. O. Kennedy, F. L. Dodd, B. C. Robertson, E. J. Okello, J. L. Reay, A. B. Scholey and C. F. Haskell, Monoterpenoid extract of sage (Salvia lavandulaefolia) with cholinesterase inhibiting properties improves cognitive performance and mood in healthy adults, J. Psychopharmacol., 2011, 25, 1088-1100.

145 K. A. Herrlinger, K. M. Nieman, K. D. Sanoshy, B. A. Fonseca, J. A. Lasrado, A. L. Schild, K. C. Maki, K. A. Wesnes and M. A. Ceddia, Spearmint extract improves working memory in men and women with ageassociated memory impairment, J. Altern. Complement. Med., 2018, 24, 37-47.

146 D. O. Kennedy, G. Wake, S. Savelev, N. T. Tildesley, E. K. Perry, K. A. Wesnes and A. B. Scholey, Modulation of mood and cognitive performance following acute administration of single doses of Melissa officinalis (Lemon balm) with human CNS nicotinic and muscarinic receptor-binding properties, Neuropsychopharmacology, 2003, 28, 1871-1881.

147 S. V. Joseph, I. Edirisinghe and B. M. Burton-Freeman, Fruit polyphenols: A review of anti-inflammatory effects in humans, Crit. Rev. Food Sci. Nutr., 2016, 56, 419-444.

148 H. Nyambe-Silavwe and G. Williamson, Polyphenol- and fibre-rich dried fruits with green tea attenuate starchderived postprandial blood glucose and insulin: a randomised, controlled, single-blind, cross-over intervention, Br. J. Nutr., 2016, 116, 443-450.

149 A. Kerimi, H. Nyambe-Silavwe, J. S. Gauer, F. A. TomasBarberan and G. Williamson, Pomegranate juice, but not an extract, confers a lower glycemic response on a highglycemic index food: randomized, crossover, controlled trials in healthy subjects, Am. J. Clin. Nutr., 2017, 106, 1384-1393.

150 S. N. Warnakulasuriya, Ziaullah and H. P. Rupasinghe, Long chain fatty acid acylated derivatives of quercetin-3-oglucoside as antioxidants to prevent lipid oxidation, Biomolecules, 2014, 4, 980-993. 
151 B. Zhou, L. M. Wu, L. Yang and Z. L. Liu, Evidence for alpha-tocopherol regeneration reaction of green tea polyphenols in SDS micelles, Free Radicals Biol. Med., 2005, $38,78-84$.

152 J. Frank, A. Budek, T. Lundh, R. S. Parker, J. E. Swanson, C. F. Lourenco, B. Gago, J. Laranjinha, B. Vessby and A. Kamal-Eldin, Dietary flavonoids with a catechol structure increase alpha-tocopherol in rats and protect the vitamin from oxidation in vitro, J. Lipid Res., 2006, 47, 2718-2725.
153 C. P. Bondonno, L. A. Downey, K. D. Croft, A. Scholey, C. Stough, X. Yang, M. J. Considine, N. C. Ward, I. B. Puddey, E. Swinny, A. Mubarak and J. M. Hodgson, The acute effect of flavonoid-rich apples and nitrate-rich spinach on cognitive performance and mood in healthy men and women, Food Funct., 2014, 5, 849-858.

154 P. H. MacArthur, S. Shiva and M. T. Gladwin, Measurement of circulating nitrite and S-nitrosothiols by reductive chemiluminescence, J. Chromatogr. B: Anal. Technol. Biomed. Life Sci., 2007, 851, 93-105. 